\title{
Infectious diseases and the air travel - a new Pandora's box?
}

\author{
Andrei Vata ${ }^{1,2}$, Larisa Miftode ${ }^{1,2}$, Maria Obreja ${ }^{2}$, Radu Miftode' ${ }^{1}$, Luminita Gina Vata ${ }^{1}$ \\ 1"Grigore T. Popa" University of Medicine and Pharmacy, Iasi, Romania \\ 2"Sfanta Parascheva" Hospital of Infectious Diseases, Iasi, Romania
}

\begin{abstract}
While traveling by plane, passengers and pilots live in a controlled, partially isolated, heated and filtered microclimate in order to provide them comfort and safety. However, due to the large number of people $/ \mathrm{m}^{3}$ of air and because of the sometimes unpredictable airflow that carry germs, the passengers present an important risk especially for infectious diseases with airborne transmission; the transmission of tuberculosis, influenza, measles, MERS, SARS, and the new SARS-2-CoV in this context has been widely proven and described. Vector-borne diseases are another threat because they can travel (especially mosquitoes) in the luggage or clothes of passengers and can spread diseases such as malaria, Chikungunya fever or yellow fever at long distances. The multi-drug resistant bacteria spreading in the case of repatriated travelers after hospitalizations abroad represents another problem. Even in the absence of a truly in-flight disease transmission, air travel nowadays represents a way to spread diseases such as Ebola, SARS or animal and wildlife-related infections between different countries or even continents. Disinfection, disinsection, the use of more efficient methods of air filtering in the cabin, the strict control of the passengers at check-in and check-out represent protecting measures against these risks, whose efficiency remains to be proven.
\end{abstract}

Keywords: epidemic, vector, contagiousness

\section{INTRODUCTION}

The flight fascinated man since ancient times see the myth of Icarus, the one punished by the gods for his courtesy to lift himself off the earth and to approach the sun. However, this has not stopped the aviation pioneers from the beginning of the $20^{\text {th }}$ century (including famous romanians like Traian Vuia or Aurel Vlaicu) to go in the sky and to lay the foundation of what we today call a modern aviation. In 2018, the airlines have transported over 4 billion passengers [1], and there is an increasing trend concerning the number of passengers in the years to come.

During the flight, passengers and cabin crew live in a controlled, partially closed, heated and fil- tered microclimate in order to provide them comfort and safety. However, sometimes, due to multiple factors (most of them difficult to control), infectious diseases can be transmitted during the flight or carried over long distances, in a short period of time. This has also been demonstrated during the latest influenza pandemic (2009) or in the case of emerging diseases such as Severe Acute Respiratory Syndrome (SARS), the Middle East Respiratory Syndrome (MERS), or the more recent Ebola, measles or SARS-CoV-2 epidemic.

The aircraft cabin is one of the areas with the smallest air volume / person found in all public spaces and thus the risk of transmitting some airborne infectious diseases such as influenza, tuberculosis, measles, SARS, MERS, meningococcal 
infection or common cold could be considerably increased. Spreading of digestive tract infections such as food poisoning with salmonella or staphylococcus, dysentery, cholera or viral enteritis were also reported, but with a lower incidence. Another possible risk of commercial flights or airport transit is represented by vector-borne diseases, with known outbreaks of malaria or Dengue fever.

Compared to the overall volume of passengers, these flight-associated infectious events represent a small but pretty significant proportion, given the severe, sometimes lethal evolution of such diseases. Most likely, their number is under-reported due to several factors such as:

- The difficulty of tracking a large number of passengers, from various countries of origin or passengers only transiting the airport for another flight.

- The long incubation period of some potentially flight-acquired infectious diseases (ID), such as tuberculosis or viral hepatitis

- The ubiquitous nature of some IDs such as common cold or food poisoning

- Failure of these patients to present to the doctor / health authority who have the competence to report the incident to the epidemiological surveillance network

\section{AIR-BORNE DISEASES}

Modern aircraft fly at an average cruise altitude of 12,000 meters (between 3,500 and 13,000 m) above sea level [3]. There, the outside temperatures range from $-30^{\circ}$ to $-70^{\circ} \mathrm{C}$, while the atmospheric pressure is about 8 times lower than the one found at ground level, and the humidity is close to zero [4]. These conditions would be difficult (if not impossible) to withstand by the human body, so the passengers, during the flight, are completely dependent on the environmental control system that maintains the pressure and also heats, humidifies and filters the air in the cabin.

Since the 1980s, especially to achieve significant fuel economy, the composition of air cabin consists of $50 \%$ air taken from outside, through the engine intake area (compressed, cooled, humidified) and 50\% inside air, recirculated and filtered. In comparison, modern office buildings use $20 \%$ outdoor air and $80 \%$ recirculated air. [5] The air circulation in the cabin is semi-automatic, divided into several sectors, and the airflow is laminar, transverse in the direction of flight, precisely to limit the risk of transmission of respiratory pathogens along the aircraft. On average, cabin air is changed 15-20 times per hour (compared to 12 times / hour for an office building) [2]. Outside air (considered sterile at cruise altitude) and recirculated cabin air are passed through special filters which are designed to eliminate any potential contamination for passengers or crew: for ozone (catalytic ozone converter), hydrocarbons (charcoal filter) and particles (True High- Efficiency Particle Filters (True HEPA) or High-Efficiency Particle Filters (HEPA).

HEPA filters retain between 85 and $99.97 \%$ of particles over $0.1 \mu \mathrm{m}$, removing dust, vapors, bacteria, fungi and most viruses from the atmosphere. They are generally replaced after 4,000-12,000 flight hours. Even if they are kept in use for a longer period of time, they do not reduce their filtering efficiency, but only decrease the volume and speed of air filtration. Particles smaller than $0.1 \mu \mathrm{m}$ are "captured" by electrostatic and brownian forces. Currently, the larger aircraft (capacity over 100 passengers) are equipped with HEPA filters (85\% of US aircraft in 2002), but smaller aircraft produced before this year benefit from such filtering only in a small proportion [2].

The risk of airborne transmitted infectious disease in the aircraft cabin is difficult to quantify and is influenced by several factors such as the type and amount of pathogen released from the source, the distance from it, the circulation of air currents, passengers and crew movements during the flight and, of course, the exposure time.

Influenza is a highly contagious disease, with only five infectious aerosol particles being sufficient for acquiring the illness.

A meta-analysis published in 2014 by the ECDC, which included 402 articles published between 1960 and 2014, found at least 15 papers which confirmed the on-board transmission of influenza viruses (A (H1N1) pdm2009, B / Beijing / 184 / 93-) like, A / Taiwan / 1/86 (H1N1) virus, A / Alaska / 18/77 (H3N2)). The flight time ranged from 45 minutes to over 20 hours and apparently the rate of infection was higher for short flights [6].

Modern cabin air circulation and filtration systems seem to significantly reduce the number of 
such incidents in recent years. The importance of the permanent functioning of the environmental control system also emerges from the 1979 event in USA [7] when a 54-passenger plane was immobilized on the ground for 3 hours due to a malfunction, during which time its systems failed to operate. In these circumstances, due to the presence of a symptomatic passenger on board, within the next 3 days $72 \%$ of the rest of the passengers developed influenza (virus A / Texas / 1 / (H3N2)).

Another recent study assessed the results from 11 publications that tracked the potential transmission of influenza A(H1N1)pdm09 during the 2009 pandemic. In total, 2,165 (51\%) of the total 4,252 flu passengers on board were monitored. There were described 163 secondary cases, the calculated attack rate being $7.5 \%$. Only $42 \%$ of the passengers in secondary cases were within a 2-lane radius of the source [8], which confirms the fear that the influenza virus may spread over longer distances in the aircraft cabin.

WHO states that the risk of on-board transmission of influenza is rather low, similar to the one to be found in other crowded environment (theaters, trains, buses, office buildings) [9].

Another potential source for the spread of influenza in the plane is represented by the crew members who, if they are working while infected, could come in contact with a large number of potentially susceptible passengers. According to a study cited by Mangili [10], the flu vaccination rate of flight crews varies only between 20 and $27 \%$.

The most effective measure to prevent influenza infection on board is the annual vaccination. Other measures designed to reduce the risk of illness are: wearing masks during flight, the strict hygiene of the hands, especially if coughing or sneezing. Starting with the emergence of neuraminidase inhibitors (especially oseltamivir), there is the possibility of using post-exposure chemoprophylaxis.

The beginning of the third millennium was marked by the unexpected emergence of some previously unknown infectious diseases, which, mainly due to air transport, spreaded rapidly - within weeks or months, on several continents, generating fears about new devastating pandemics. If in the case of the first two such diseases - severe respiratory syndrome (SARS) and the Middle East respiratory syndrome (MERS) - the things were not so dramatic it is because of the pathogen characteristics (hard to transmit) and of the health professionals who have managed well these diseases.

The first cases of SARS were reported to the WHO by China in November 2002, the virus being identified in Germany a few weeks later. The disease was initially accidentally exported to Hong Kong by a doctor who was activating in China, with more than 24 countries reporting cases in just a few months. The disease has been shown to be airborne transmitted, through large drops and probably by aerosols, as evidenced by the incident in the Amoy Gardens apartment block in Hong Kong, where, in March 2003, 321 infection cases of some tenants from different floors were recorded, due to shared ventilation pipes in the bathroom. A month earlier, during a 3 hour flight between Hong Kong and Taipei in a Boeing 737-300 aircraft (February 2003), with 120 passengers on board, one single patient was responsible for 22 secondary cases, in a range up to 6 rows away from him.

The spread of the disease through air transport and in-flight transmission were further documented: 40 flights were suspected of carrying SARS patients, and 5 were associated with the transmission of the disease to another 37 (most of them were placed at a distance of up to 5 lines from source) [2].

Fortunately, the epidemic was extinguished by the end of 2003, totaling 8,096 probable cases, of which 774 deaths (fatality rate of 9.6\%) [11].

The first known cases of MERS appeared in 2012 after the death of a sheikh in Saudi Arabia; from his sputum a new pathogenic human coronavirus was isolated. The second case was confirmed in a patient from Qatar a few weeks later, then other cases were reported in Jordan and other countries in the region. The disease spreaded rapidly in 27 countries in Europe, USA, Africa and Asia, by air transport of patients with known disease or during incubation. Fortunately, the virus is not easily transmitted between humans, and close contact is required (numerous nosocomial infections are known). However, in 2015 a regional epidemic was triggered in South Korea by a traveler who had returned from Saudi Arabia and generated 186 diseases and 36 deaths. Up to date, there are no known cases of in-flight transmission. The number of confirmed cases reached 2,468 in September 2019, of which 851 died [12]. 


\section{New threats - The new 2019 Coronavirus (SARS- 2-CoV)}

Since the last day of December 2019, the Chinese city of Wuhan has reported an outbreak of atypical pneumonia caused by a new zoonotic coronavirus (SARS-2-CoV). By March 10, 2020, 114,573 cases of SARS-2-CoV infections have already been confirmed, of which 4,029 deaths in 115 countries and territories.

In the study by Wu et al. [13], the baseline reproduction number for SARS-2-CoV was 2.68, while WHO estimated a transmissibility rate between 1.4 and 2.5, and Zhao et al. [14] - 3.58. In comparison, the transmissibility rate for SARS was 2.0, and for the common cold it was 1.3.

Case definition was proposed by the Chinese Center for Disease Control and Prevention (CDC). A suspicious or probable case is defined as a case that meets: (1) three clinical criteria or (2) two clinical criteria and one epidemiological criteria.

The clinical criteria are:

- fever;

- radiographic evidence of pneumonia or acute respiratory distress syndrome;

- leukopenia / normal values of leukocytes or lymphopenia

The epidemiological criteria are:

- Wuhan residents / travel history in Wuhan or other areas with confirmed virus transmission within 14 days before the onset of symptoms;

- contact with patients with fever and symptoms of respiratory infection within 14 days before the onset of symptoms;

- connection with any confirmed cases or groups of suspected cases.

In the study conducted by Guan WJ et al. [15], which analyzed 1,099 patients with SARS-2-CoV confirmed infection, the following characteristics were highlighted:

- Average incubation time was 3.0 days (2-14 days)

- Symptoms: fever (87.9\%), cough (67.7\%), vomiting (5.0\%), diarrhea (3.7\%)

- $76.4 \%$ had pneumonia

- $84.1 \%$ had lymphopenia and $36.2 \%$ thrombocytopenia

- 3.4\% developed acute respiratory distress syndrome, $38.0 \%$ required oxygen, $5.0 \%$ re- quired admission to an intensive care unit and $1.4 \%$ died.

The mortality rate varies according to age $(14.8 \%$ - in patients over 80 years, while among patients under 9 years there have been no deaths so far), gender (male $-2.8 \%$, female $-1.7 \%$ ), associated pathologies (cardiovascular $-10.5 \%$, diabetes $-7.3 \%$, chronic respiratory diseases $-6.3 \%$ ) [16].

A coronavirus vaccine could prevent infections and stop the spread of the disease. After the 2003 SARS outbreak, researchers needed about 20 months to prepare a vaccine for human studies. With the outbreak of Zika in 2015, the vaccine development timeline has been reduced to six months. Researchers have already studied the genome of the new coronavirus and identified proteins that are crucial for infection. Scientists from the National Institutes of Health in Australia and at least three companies are working on this vaccine.

Additional information on SARS-2-CoV is needed to understand the transmission, severity of the disease and risks to the general population, but air transport is certainly responsible for the rapid spread of the virus in Europe, North America and other Asian countries.

Common cold is another disease with easy respiratory transmission, but investigating the risk of airborne transmission is difficult because of the ubiquity of the disease. One study compared the risk of disease occurrence by using in-flight recycled air $50 \%$ vs. $100 \%$ outdoor air and found no differences between the two ventilation modes [17]

$N$. meningitidis infection can have devastating consequences for meningeal or systemic disease, and airway transmission, especially in cases of human agglomeration, is known. The increased frequency of this disease in sub-Saharan Africa makes flights in this region at increased risk. Fortunately, such transmission is rare so far. At least 26 situations are known in which patients with meningococcal infection have flown during the disease or it's period of contagiousness and after which no secondary cases have been described. A flight of more than 14 hours between Los Angeles and Sydney is the only one in which a passenger transmitted a type B meningococcus to another passenger seated 12 rows apart (one was traveling a lot inside the plane, the other was sitting on the aisle) [10]. In case of rapid identification of the source of 
infection, antibiotic prophylaxis is recommended for passengers placed within a radius of at least 2 seats / rows.

Measles is a highly contagious disease, potentially lethal, with airborne transmission, in which patients are contagious 5-7 days before the onset of typical symptoms. Worldwide the number of cases is increasing in the last years - in 2019 it is estimated that there were 3 times more illnesses compared to 2018, and the developed countries were also affected - 13,264 cases reported in the last 12 months in the EU. WHO estimates that measles was responsible for 110,000 deaths in 2017 [18].

There are numerous reports of measles transmission at the aircraft cabin level. Until a few years ago, most cases of measles from the US or Australia were imported from developing countries and it is estimated that $30 \%$ of patients flew while they were symptomatic. Two secondary cases are known after a patient's flight to Brazil, 2 incidents of the same type in the Netherlands (2007). In Australia, 45 cases were described as acquired in flight between 2007 and 2011 (49 flights were investigated - 13 domestic and 36 international) [10]. In the USA, between 2008 and 2011, 74 patients were known to have traveled by airplane while being contagious, in 108 flights; the subsequent investigation followed 2,673 of the 3,399 (79\%) connected cases from the planes, being identified 9 secondary cases. Even in Finland, the country where the vaccine coverage is $95 \%, 47$ cases were acquired during the plane trip between 1996 and 2012.

Due to the high degree of contagiousness of the disease, the infectious contact can be made during the transit of the airport (check-in counter, security control, waiting room), and the best way of protection is the presence of anti-measles antibodies obtained through vaccination (or passing through the disease).

The Ebola epidemic (EVD) between 2014 and 2016 in 3 states in western Africa (Guinea, Sierra Leone, Liberia) was the most extensive since the virus was discovered in 1976 , with 28,652 probable or confirmed cases and 11,315 deaths. In the 2018's summer, the disease re-emerged in another African country - the Democratic Republic of Congo, and this epidemic is ongoing, with 3,298 cases and 2,196 deaths so far (November 2019). [19] The inter-human transmission of the disease is still in- completely understood, but it involves close contact with the patient, blood or other biological fluids, contact with the skin or mucous membranes. The only subtype of airborne virus known so far is Reston (responsible for a milder form of EVD).

There are several studies that have attempted to determine the potential for EVD transmission by air transport. Bogoch et al. [20] estimated that, during the 2014-2016 epidemic, on average 2.8 infected patients left their country of origin each month, most of them leaving to other developing countries (most commonly Ghana and Senegal), not so well prepared to deal with the outbreak of a local epidemic. The risk of exporting patients to Europe (England and France) was considered 8 times higher than for the USA [20].

EVD patients were exported from Africa, the majority being medical personnel evacuated to be treated in their country of origin - 22 such cases are known. Three cases of nosocomial infections occurred outside Africa, in the medical staff who cared for these patients (2 US cases, 1 Spain case) [21]. Fortunately, so far there have been no cases of EVD transmitted during the flight.

International institutions (WHO, ECDC) have developed strategies to reduce the risk of transmission of the disease through or during the air travel, which include information of travelers, international collaboration of health authorities, control of passengers when leaving the epidemic area (which seems most efficiently [20]) or upon arrival in the country of destination.

Tuberculosis is a contagious disease transmitted through Pflugge drops, being one of the first 10 causes of death globally. It is estimated that a quarter of the world's population has been infected with M. tuberculosis, and in 2018 around 10 million people have developed the disease. [22]

There are numerous known cases of exposure to tuberculosis on board of an airplane, but cohort studies are rare due to the difficulties of long-term follow-up of the subjects (long incubation period), differences in the definition of tuberculosis and the problems of active infection diagnosis.

The studies carried out at the end of the 20th century were those that established that the maximum risk of infection lies within a 2-rows radius compared to the source passenger, especially in flights with a duration of over 8 hours [2]. 
Two of 7 studies from the 1990s found evidence of flight-related transmission: a sick stewardess resulted in 2 tuberculin test conversions from 212 colleagues and 59 frequent travelers, followed for 5 months [25]; after a flight from Baltimore to Chicago and then to Honolulu 4/15 passengers who were 2 rows away from the patient had conversion of intradermoreaction to tuberculin [26].

Abubakar's (2010) meta-analysis which included 13 publications that investigated 53 flights with 38 active tuberculosis passengers (1 rifampicin-resistant, 1 isoniazid-resistant, 4 MDR TB, 1 XDR TB), following 2761 passengers and crew members, found 10 cases of IDR positive for tuberculin, but no cases of secondary active tuberculosis [24].

A study based on data from an airline company, estimated that the prevalence of tuberculosis travelers is 0.05 per 100,000 ( 0.36 for flights from Africa and 0.35 for flights from India). When traveling with a symptomatic patient, an average infection risk of 1:1,000 was found by Keyton [26], a risk similar to the one found in other confined spaces.

\section{DISEASES WITH VECTOR TRANSMISSION}

Malaria is the most widespread parasitic disease in the world with serious individual, public health and economic consequences, being usually associated with tropical areas around the globe. However, now it is recognized a so-called "airport malaria" - a disease acquired while staying at the airport (or during the flight), occurring in people who have not visited endemic areas (in the last 12 months). It is transmitted by Anophel mosquitoes, clandestine passengers in the cabin or luggage compartment of aircraft with origin or stops in endemic areas. Under favorable temperature conditions they survive for a time in airports and neighboring areas, managing to transmit the disease to travelers or the resident population. The area the mosquito can travel through is $7-15 \mathrm{~km}$ around the airport, but the area can be larger if carried in luggage [30].

Fortunately, malaria acquired this way is rare, with few cases reported in recent years. An older study (1969-1999) estimated that the average frequency of these cases is 2 / year [24]; In Europe, 89 cases of airport malaria have been reported in several areas [30-33]. Gratz [29] estimated that in
1994 between 2,000 and 5,000 Anophel mosquitoes were imported into France (8-20 / flight from endemic areas).

The disease can be difficult to recognize and diagnose in the absence of epidemiological information and can have severe evolution and consequences for the traveler. Another concern is the introduction of the parasite with anti-malarial resistance in areas previously without this issue.

Other traditional "tropical" diseases, such as dengue fever, yellow fever, Chikungunya, Zika, could be transmitted by plane and around the airport by native Aedes mosquitoes. Their contact with travelers already infected in endemic areas could be the beginning of local epidemics, in temperate areas. Jelinek [35] described in 1998 the case of two German tourists probably infected at the airport with the dengue virus and who developed the disease in their native country.

WHO has recommended [36] the application of measures to disinsect the interior of airplanes with origin in endemic areas (between flights) and airport spaces and its surroundings, but they are not applied by all countries. There are also fears that disinfection of aircraft will not solve the problem of the spread of vector-transmitted diseases by plane flight [37].

\section{DISEASES WITH DIGESTIVE TRANSMISSION}

In recent years there have been no reported infections with digestive transmission during the flight, especially due to the improvement of food preservation and handling, the use of pre-packaged foods, but such situations have existed in the past and there is a risk that things will be repeated.

The most common pathogen involved in foodborne infections with origin on board commercial flights is Salmonella; between 1947 and 1999, over 4000 infected passengers and 11 deaths were registered. Staphylococcus aureus was also involved in at least 11 incidents, resulting in 1 death [2].

There are known cases where cholera was transmitted inside the plane: in 1975 during the flight from London to Sydney via Singapore, through the consumption of cold appetizers contaminated with Vibrio cholerae (47 patients, 1 death) or in 1992 flight originating in Latin America, after eating a seafood menu (75 patients, 1 death) [2] 
Viruses have been less involved in such incidents, but a case is known where transmission of norovirus was possible after consumption of contaminated orange juice.

\section{BACTERIAL RESISTANCE TO ANTIBIOTICS AND TRAVEL BY PLANE}

WHO has recently recognized that the spread of bacterial resistance to antibiotics is one of the greatest threats to public health, food security and global development [38]. Although bacterial resistance exists on all continents, some areas, especially in developing countries, are more affected by this problem. The exchange of resistant bacteria from geographically distant regions can be now easy and fast due to air travel.

A recent study found that $64 \%$ of tourists in India are colonized with ESBL-producing enterobacterial strains (34\% from Africa). The presence of diarrheal disease during the travel, administration of antibiotics or hospitalization abroad are cited as risk factors for the acquisition of these bacteria. Fortunately, $75 \%$ of them will get rid of these strains after 6 months of return [39], but during this time they can spread these bacteria in the community of origin or in hospitals.

The case of an outbreak of MRSA infection in a neo-natology unit in England is known to have originated from a nurse who had brought the strain from a recent trip to the Philippines [40].

New Delhi metallo-beta-lactamase 1, an enzyme that provides resistance to most beta-lactam antibi-

\section{REFERENCES}

1. https://www.statista.com/statistics/564717/airline-industry-passengertraffic-globally/.

2. Mangili A, Gendreau MA. Transmission of infectious diseases during commercial air travel. Lancet 2005; 365:989-96.

3. Giaconia C, Orioli A, Di Gangi A. Air quality and relative humidity in commercial aircrafts: An experimental investigation on short-haul domestic flights. Building and Environment 2013; 67:69-81.

4. National Research Council (US) Committee on Air Quality in Passenger Cabins of Commercial Aircraft. The Airliner Cabin Environment and the Health of Passengers and Crew. Washington (DC): National Academies Press (US); 2002, 34-36.

5. DeHart RL. Health Issues of Air Travel. Annual Review of Public Health 2003; 24(1):133-151.

6. European Centre for Disease Prevention and Control. Risk assessment guidelines for infectious diseases transmitted on aircraft (RAGIDA) - Influenza. Stockholm: ECDC; 2014

7. Moser MR, Bender TR, Margolis HS, Noble GR, Kendal AP, Ritter DG. An outbreak of influenza aboard a commercial airliner. Am J Epidemiol 1979; 110(1):1-6. otics - including carbapenems, was initially detected in a $K$. pneumoniae strain isolated from the urine of a Swedish patient who had been transferred from an Indian hospital. Subsequently, it appeared and spread to many hospitals in Europe and America, both in $K$. pneumoniae strains and in other bacteria (E. coli, Acinetobacter spp.) [41, 42].

So far, there are no cases of transmission of resistant multidrug bacteria on board commercial aircraft, but such a situation is plausible, given the volume of passengers and the close contact between them in the cabin.

\section{CONCLUSIONS}

Modern aviation is one of the most regulated, supervised and safe public modes of transport, equipped with multiple means of combating infectious risks - from air conditioning and filtration, to disinsection and the way of preparing, storing and serving food.

Even though, after the discovery and development of antibiotics, some predicted the disappearance of infectious diseases, today's reality shows that this wish cannot be achieved in the near future. Today's air transport mobilizes over a long distance and in a short time an impressive number of people, who can sometimes carry infectious pathogens. Thus, when we talk about infectious diseases with epidemic potential today, we must do it from a global perspective, the political borders and distances being only relative obstacles in the way of spreading these diseases.

Conflict of interest: none declared Financial support: none declared

8. Leitmeyer K, Adlhoch C. Influenza Transmission on aircraft. A systematic literature review. Epidemiology 2016; 27(5):743-751.

9. https://www.who.int/ith/mode_of_travel/tcd_aircraft/en/.

10. Mangili A, Vindenes T, Gendreau M. Infectious risks of air travel. Microbiol Spectrum 2015; 3(5):IOL5-0009-2015.

11. WHO. Summary of probable SARS cases with onset of illness from 1 November 2002 to 31 July 2003. http://www.who.int/csr/sars/country/ table2004_04_21/en/.

12. WHO. MERS situation update September 2019. https://www.who.int/ emergencies/mers-cov/en/.

13. Wu J, Leung K, Leung GM. Nowcasting and forecasting the potential domestic and international spread of the 2019-nCoV outbreak originating in Wuhan, China: A modelling study. The Lancet 2020 https://doi.org/10.1016/S0140-6736(20)30260-9.

14. Zhao S, Lin Q, Ran J et al. Preliminary estimation of the basic reproduction number of novel coronavirus (2019-nCoV) in China, from 2019 to 2020: A data-driven analysis in the early phase of the outbreak. Int J Infect Dis 2020; 92:214-217. 
15. Guan WJ, NI YZ, Hu Y et al. Clinical characteristics of 2019 novel coronavirus infection in China. medRxiv 2020.

16. The Novel Coronavirus Pneumonia Emergency Response Epidemiology Team. Vital Surveillances: The Epidemiological Characteristics of an Outbreak of 2019 Novel Coronavirus Diseases (COVID-19) - China, 2020. China CDC Weekly 2020; 2(8):113-122.

17. Zitter JN, Mazonson PD, Miller DP, Hulley SB, Balmes JR. Aircraft cabin air recirculation and symptoms of the common cold. JAMA 2002; 288:483-486.

18. WHO - Measles fact sheet. https://www.who.int/news-room/ fact-sheets/detail/measles.

19. WHO. Ebola Virus Disease Democratic Republic of Congo: External Situation Report 68 / 2019. https://apps.who.int/iris/rest/ bitstreams/1261378/retrieve.

20. Bogoch II, Creatore MI, Cetron MS et al. Assessment of the potential for international dissemination of Ebola virus via commercial air travel during the 2014 west African outbreak. The Lancet 2015; 385(9962):29-35

21. Cowling BJ, Yu H. Ebola: Worldwide dissemination risk and response priorities. The Lancet 2015; 385(9962):7-9.

22. WHO. Global tuberculosis report 2019. https://apps.who.int/iris/ bitstream/handle/10665/329368/9789241565714-eng.pdf?ua=1

23. Ko G, Thompson KM, Nardell EA. Estimation of tuberculosis risk on a commercial airliner. Risk Anal 2004; 24: 379-388.

24. Abubakar I. Tuberculosis and air travel: A systematic review and analysis of policy. The Lancet Infectious Diseases 2010; 10(3):176183.

25. Driver CR, Valway SE, Morgan WM, Onorato IM, Castro KG. Transmission of Mycobacterium tuberculosis associated with air travel. JAMA 1994; 272: 1031-1035.

26. Kenyon TA, Valway SE, Ihle WW, Onorato IM, Castro KG. Transmission of multidrug resistant Mycobacterium tuberculosis during a long airplane flight. N Engl J Med 1996; 334:933-938.

27. Byrne N. Low prevalence of TB on long-haul aircraft. Travel Med Infect Dis 2007; 5:18-23.

28. Tatem AJ, Rogers DJ, Hay SI. Estimating the malaria risk of African mosquito movement by air travel. Malaria Journal 2006, 5:57.

29. Gratz NG, Steffen R, Cocksedge W. Why aircraft disinsection? Bull World Health Organ 2000; 78(8):995-1004.

30. Thang HD, Elsas RM, Veenstra J. Airport malaria: Report of a case and a brief review of the literature. Neth J Med 2002; 60:441-443.
31. Van Den Ende J, Lynen L, Elsen $P$ et al. A cluster of airport malaria in Belgium in 1995. Acta Clin Belg 1998; 53:259-263.

32. Majori G, Gradoni L, Gianzi FP, Carboni P, Cioppi A, Aureli G. Two imported malaria cases from Switzerland. Tropl Med Parasitol 1990; 41:439-440.

33. Rodger AJ, Cooke GS, Ord R, Sutherland CJ, Pasvol G. Cluster of falciparum malaria cases in UK airport. Emerg Infect Dis 2008; 14:1284-1286.

34. Isaäcson M. Airport malaria: A review. Bull World Health Organ 1989; 67:737-743.

35. Jelinek T, Dobler G, Nothdurft H. Evidence of dengue fever virus infection in a German couple returning from Hawaii. J Travel Med 1998; 5:44-45.

36. WHO. International health regulations (2005) -- 3rd ed. http://apps. who.int/iris/bitstream/10665/246107/1/9789241580496-eng. pdf?ua=1.

37. Mier-Y-Teran-Romero L, Tatem AJ, Johansson MA. Mosquitoes on a plane: Disinsection will not stop the spread of vector-borne pathogens, a simulation study. PLoS Negl Trop Dis 2017; 11(7):e0005683.

38. WHO. Antibiotic resistance. https://www.who.int/news-room/ fact-sheets/detail/antibiotic-resistance.

39. Schwartz KL, Morris SK. Travel and the spread of drug-resistant bacteria. Curr Inf Dis Rep 2018; 20:29.

40. Ali $H$, Nash JQ, Kearns AM et al. Outbreak of a south West Pacific clone Panton-Valentine leucocidin-positive methicillin-resistant Staphylococcus aureus infection in a UK neonatal intensive care unit. J Hosp Infect 2012; 80:293-298.

41. Solovăstru GL, Stîncanu A, De Ascentiis A, Cappare G, Mattana P, Vâță D. Randomized, controlled study of innovative spray formulation containing ozonated oil and $\alpha$-bisabolol in the topic treatment of chronic venous leg ulcers. Adv Skin Wound Care 2015;28(9):406-9.

42. Yong D, Toleman MA, Giske CG et al. Characterization of a new metallo- $\beta$-lactamase gene, blaNDM- 1 , and a novel erythromycin esterase gene carried on a unique genetic structure in Klebsiella pneumoniae sequence type 14 from India. Antimicrob Agents Chemother 2009; 53(12):5046-5054. 